\title{
Presupuestos Participativos (PPs) e Instituciones Participativas (IPs) en Brasil: Criterios (y marco) para la evaluación de experiencias y casos ${ }^{1}$
}

\author{
Valdemir Pires \\ Universidade Estadual Paulista, Brasil \\ vapires@terra.com.br \\ Carmen Pineda Nebot \\ Consultora de Administraciones Públicas \\ carmenpinedanebot@hotmail.com
}

Recibido: 16 de mayo de 2013

Aceptado: 24 de mayo de 2013

\begin{abstract}
Resumen
En este artículo se identifican diversos criterios relevantes para la evaluación de los presupuestos participativos y las instituciones participativas que son adoptados actualmente por gobiernos tanto de Brasil como de otros lugares del mundo, destacando la importancia de una evaluación global que integre todos estos criterios. Finalmente, se recomienda prestar especial atención a la evaluación de la deseabilidad de esas instituciones, que no siempre son percibidas positivamente por las teorías sobre la democracia.
\end{abstract} públicas.

\section{Participatory budgets (PBs) and participatory institutions (PIs) in Brasil: criteria (and framework) for the evaluation of experiences and cases}

\begin{abstract}
This paper identifies relevant criteria for evaluation of participatory budgeting and participatory institutions that are currently adopted by governments of both Brazil and elsewhere, highlighting the importance of a comprehensive assessment that integrates all these criteria. Finally, it is recommended to pay special attention to assessing the desirability of these institutions, which are not always positively for theories on democracy.
\end{abstract}

Key words

Participatory democracy, participatory institutions, participatory budgeting, evaluation of public policies. 


\section{INTRODUCCIÓN}

A lo largo del período comprendido entre las dos últimas décadas del siglo XX y la primera década del siglo XXI, Brasil pasó por profundas transformaciones sociales y políticas. Saliendo de un régimen dictatorial-militar que duro de 1964 a 1985, el país retornó a la democracia gracias a las grandes movilizaciones populares y sindicales, cambió su Constitución (1988), amplió los derechos políticos y sociales, impulsó reformas del Estado y cambió el modus operandi de las administraciones públicas, haciéndolas (aunque no suficientemente) más transparentes y permeables a los intereses ciudadanos (Dagnino, 2002; Bresser-Pereira; Wilheim; Sola, 1999). La participación ciudadana, a pesar de que inicialmente fue prohibida por razones de seguridad nacional, fue intensa en el período de transición de la dictadura a la democracia y después, una vez consolidado el nuevo régimen, se convirtió, primero, en una práctica cotidiana de las poblaciones urbanas en su relación con los poderes constituidos y, en un segundo momento, en un derecho incluido en la Constitución y en las leyes federales, de los estados y municipales.

Las experiencias de presupuesto participativo (PPs) estuvieron entre las experiencias pioneras de participación ciudadana en los procesos de decisión de los gobiernos, seguidas de otras instituciones participativas (IPs)2 ${ }^{2}$, como los consejos gestores municipales de políticas públicas (CGMPPs). Las IPs, en general (entre las cuales se incluyen no sólo los CGMPPs, sino también los planes directores municipales participativos, las comisiones tripartitas, las audiencias públicas y las conferencias nacionales ${ }^{3}$ ), y los PPs, como un tipo específico y conocido de IP, se convierten en el referente brasileño de poco más de dos décadas de innovación institucional de cuño democrático. Comenzando ahora a ser cuestionadas y evaluadas bajo parámetros cada día más rigurosos, quizá porque pasada la fase eufórica de su implementación, el ánimo participativo se enfrió y las promesas no cumplidas y las expectativas frustradas se van acumulando.

En un contexto de cuasi consenso, tanto político como académico, en torno al protagonismo ciudadano y a la participación ciudadana en las decisiones del gobierno y en la gestión de políticas públicas, mantenido en medio de un clima de baja tendencia de los individuos a la implicación en los asuntos de los gobiernos, de los partidos, sindicales o electorales, el foco de las evaluaciones de las IPs incide sobre su efectividad (Avritzer, 2011), justamente a causa de la distancia que muchas veces se da en estos temas entre el discurso y la práctica.

A pesar del acierto que supone prestar atención a la efectividad en la evaluación de las IPs, es muy importante considerar también otras dimensiones, como la eficiencia, la eficacia, la sostenibilidad, la replicabilidad y la deseabilidad. Es sobre esos dife-

2 La expresión “instituciones participativas” es utilizada, aquí, en el sentido utilizado por Avritzer (2008): "formas diferenciadas de incorporación de ciudadanos y asociaciones de la sociedad civil en la deliberación sobre políticas."

3 No se consideran aquí el plebiscito, el referéndum y la iniciativa popular (previstos en la Constitución brasileña de 1988) porque, a pesar de ser importantes conquistas, han sido poco utilizados. 
rentes puntos de la evaluación de los PPs e IPs en los que se centra este trabajo, dividido en tres partes. Después de un breve comentario sobre la evolución en las tres últimas décadas de los PPs e IPs en Brasil, (sección 1), se tratan cada uno de los puntos de evaluación citados anteriormente (sección 2), así como una visión integrada de los mismos (sección 4), deteniéndose en el análisis de la dimensión de deseabilidad, a fin de emprender un debate teórico relevante (entre adeptos de la public choice y del neorrepublicanismo), que hace posible la contraposición de dos visiones opuestas del mundo sobre la democracia, una defendida por economistas, y la otra, preferida por los especialistas políticos.

\section{OLAS DE PPS E IPS EN BRASIL MOVIÉNDOSE DEL SIGLO XX AL SIGLO XXI}

Las investigaciones más importantes señalan, desde el momento en que se inició en Porto Alegre 4 , la siguiente evolución en el número de PPs en Brasil: 13 en el mandato municipal de 1989-1992, 53 en el de 1993-1996 y 140 en el de 1997-2000 (Ribeiro y Grazia, 2003); 190 en el de 2001-2004 y 201 en el siguiente (2005-2008), según Avritzer y Wampler (2008). Esos números son aproximativos, ya que existen variaciones metodológicas y de procedimiento en los casos identificados, con diferencias en los resultados y en el efecto. No ha sido posible un estudio minucioso, que pudiera averiguar la validez de las declaraciones de los protagonistas ${ }^{5}$ ( $y$, por tanto, interesados) en las que se basaron las investigaciones, o utilizar análisis documentales, teniendo que trabajar con esos números y confiar más en otros, menores, relacionados a experiencias ampliamente difundidas, conocidas y estudiadas, tomando como paradigmáticas la pionera, de Porto Alegre (RS) y la de Belo Horizonte (MG) (diferente, pero bastante activa, como prueban los estudios realizados), o incluso las 20, señaladas por Costa (2010), que se han mantenido por lo menos tres mandatos consecutivos o alternos.

Numerosos casos de PPs menos conocidos que los identificados por Costa (2010) fueron ya objeto de estudio en programas de posgrado en Brasil ${ }^{6}$, facilitando así el poder realizar un mapa de las experiencias, pero no haciendo siempre posible evalua-

4 Antes de Porto Alegre, según Pires (2001), existieron pocas ciudades brasileñas que pusieran en marcha experiencias semejantes al Presupuesto Participativo (PP) aunque con poca repercusión, menores resultados y horizontes temporales más cortos. Podrían llamarse casos precursores del PP. Entre ellos están, Vila Velha (ES), Piracicaba (SP), Lajes (SC) y Boa Esperança (SC).

5 Torres y Grazia (2003) aplicaron cuestionarios a 103 agentes implicados en experiencias autodenominadas Presupuesto Participativo, de las inicialmente 140 identificadas, referentes al período 1997-2000. Ellas advierten que la investigación fue realizada teniendo también en cuenta una "dimensión-proyecto" del PP, que impide una lectura totalmente libre de las respuestas obtenidas. También informan que los números referentes a los dos mandatos municipales anteriores fueron obtenidos por búsquedas del Fórum Nacional de Participación Popular en las Administraciones Municipales, que es un grupo de personas, con cargos en gobiernos locales, interesadas en ampliar y consolidar el PP en Brasil. Avritzer y Wampler (2008) tuvieron oportunidad de realizar un trabajo más minucioso (al manejar un banco de datos previo, más sólido y consistente y al utilizar procedimientos del tipo snow-balling con gobiernos e instituciones, aprovechándose del material acumulado en investigaciones anteriores), pero aun así trabajaron con cuestionarios.

6 Pires y Martins (2011) identificaron casi tres decenas de trabajos en programas brasileños de posgrado. 
ciones más profundas, ya que las metodologías y las bases empíricas utilizadas en las investigaciones son muy heterogéneas y no siempre de confianza, dependiendo de lo que se quiera evaluar.

La literatura brasileña más reciente sobre IPs se ha dedicado más al estudio de los CMGPPs, debido a su difusión por todo el país y a su continuidad asegurada por ley y, también, debido a la disminución del ímpetu expansionista de los PPs. Los Consejos de Salud y de Educación son los que más interesan a los investigadores (un ejemplo de ello son: Carvalho, 1995; Gohn, 2001; Tatagiba, 2002; Guimarães, 2009; Vaz, 2011). Según la Investigación de Informaciones Básicas Municipales (MUNIC), realizada por el Instituto Brasileño de Geografía y Estadística (IBGE), los Consejos de Salud existen en el 100\% de los municipios brasileños, mientras que los de Educación y Asistencia Social se encuentran en el $90 \%$.

Los PPs y los CMGPPs son las principales modalidades de IPs utilizadas en Brasil en las últimas tres décadas. Es interesante observar que los PPs ganaron espacio durante la redemocratización del país, formando parte de una ola (la primera) de innovaciones "movimentalistas" (originadas por la movilización popular del período 19751990), mientras que los CMGPPs se fortalecieron un poco después de la Constitución de 1988, con el país ya democratizado y bajo presión por la reforma del estado (segunda ola), adquiriendo un carácter más “gerencialista” (Pires, 2009). Los diferentes contextos en los que surgieron los PPs y los CMGPPs y las distintas concepciones político-administrativas que los impulsaron son elementos fundamentales que deben tenerse en cuenta cuando se desean evaluar las prácticas correspondientes a unos y otros. No es casualidad que los CMGPPs nacieran por ley y que los PPs se pusieran y se pongan en marcha por autorregulación: la naturaleza y el grado de institucionalización de unos y otros explica en buena medida la prominencia de una lógica "movimentalista" (en declive) y de una lógica gubernamental (en ascenso).

Antes de pasar a la discusión de los aspectos posibles y deseables para evaluar las IPs en Brasil, es conveniente señalar nuestra posición en lo que respecta a su evolución. Al evaluar la gran cantidad de innovaciones institucionales, conseguidas en estos años por Brasil, que contribuyeron a la transición democrática y que contribuyen o pueden contribuir a la consolidación de la democracia y a la mejora de la gestión pública, es fundamental que no se pierda de vista que sin las prácticas participativas adoptadas por los ciudadanos y las organizaciones de la sociedad civil a lo largo de las tres últimas décadas, a pesar de sus eventuales fracasos en temas específicos y aislados, la forma social, política e institucional brasileña sería otra, probablemente mucho peor. Las metodologías y proyectos de evaluaciones que no tengan en cuenta este hecho corren el riesgo de, en el afán de comparar causas y efectos de modo supuestamente científico, identificar solamente casos de éxito y fracaso en una trayectoria exitosa en su conjunto, en cuanto que cambió en profundidad el tipo de relación entre los gobiernos y las administraciones públicas y los ciudadanos, electores y contribuyentes.

Además de la precaución de apreciar las conquistas históricas del conjunto (per262 ceptibles, pero no medibles), y no sólo los resultados inmediatos y científicamente 
comprobables, las evaluaciones de los PPs y de las IPs necesitan, para contribuir al avance del conocimiento y para la mejora de las experiencias, tener en cuenta también que tratan con una sociedad dinámica, en la que los individuos y los grupos se orientan por ideas hegemónicas y contra-hegemónicas que crean lo nuevo de modo incontrolable y muchas veces insondable y, por tanto, difícilmente analizable en cuanto esto nuevo no se convierte en relativamente antiguo, asentado. Las IPs innovadoras raramente lo son, pues transitan en el devenir difícilmente captado por análisis, aproximaciones, metodologías y evaluaciones que no se dejan penetrar por la visión dialéctica de la realidad.

\section{LOS PPS Y LAS IPS: LOS ENFOQUES DE LA EFICIENCIA, LA EFICACIA, LA EFECTIVIDAD, LA SOSTENIBILIDAD, LA REPLICABILIDAD Y LA DESEABILIDAD PARA SU EVALUACIÓN}

Los PPs y los CGMPPs son las modalidades de IP más utilizadas en la administración pública brasileña y las evaluaciones de esas experiencias son las que más pueden contribuir a la comprensión de las IPs en Brasil y a la mejora de las prácticas evaluativas de esas innovaciones institucionales tenidas como prometedoras.

Cualquier evaluación que se lleve a cabo tiene, necesariamente, una finalidad y es hecha por actores interesados en ella por diferentes razones. De ese modo, el criterio escogido en una evaluación específica tendrá siempre la marca de las condiciones bajo las cuales fue o será realizada. Una agencia de desarrollo evaluará para saber si debe o no continuar apoyando una experiencia con participación, exigida por ella como condición para financiar proyectos; partidos, políticos y electos evaluarán para calcular las ventajas y desventajas obtenidas con los mecanismos de participación que han puesto en marcha; una ONG interesada en impulsar relaciones solidarias en comunidades necesitadas podrá querer evaluar los cambios de comportamiento propiciados por un proyecto de construcción de viviendas por medio de la ayuda solidaria de la comunidad, etc. Según el objetivo de la evaluación y la visión del proceso evaluativo que tenga quien evalúa variará el criterio y la metodología de evaluación. Es importante, sin embargo, que en todos los casos se tenga una visión de conjunto de los criterios posibles. Cada uno de ellos es presentado en las subsecciones siguientes y posteriormente en conjunto, de forma integrada, en la sección 4.

\subsection{Realidad de los PPs y los CGMPPs}

No todas las experiencias autoincluidas o difícilmente identificadas como pertenecientes al universo de las IPs o como PPs, disfrutan de la básica condición de realidad. En un contexto de debate, en el que una idea es ampliamente aceptada, raros son los que la atacarán7, exponiéndose a la perdida de espacio, apoyo o votos. Mien-

7 Pires y Silva (2004) constataron en 1999 -año de gran divulgación del PP en Brasil y en el mundoel apoyo verbal que le daban numerosos políticos, de los más variados colores ideológicos; a pesar de ese 
tras que organismos multilaterales, ONGs y medios de comunicación convenzan a los electores de que los PPs y las IPs son el camino para una democracia más amplia, para gobiernos más eficientes y políticas públicas inclusivas, los electos dirán estar comprometidos con ellos hasta que no se demuestre lo contrario y muchos fingirán en la práctica algo próximo o similar, continuando hasta que la ola de credibilidad pierda impulso ${ }^{8}$. Por tanto, al considerar las dimensiones de eficiencia, eficacia, efectividad, sostenibilidad, replicabilidad y deseabilidad, la evaluación de las IPs y de los PPs debe, previamente, descartarse todo lo que no se incluya en el principio de realidad o veracidad, que no tenga apariencia de realidad en las relaciones entre el gobierno y la sociedad civil. Tarea no siempre fácil, pues la capacidad de simulación y la facilidad para obtener apoyo son bastante frecuentes en contextos políticos atrasados en lo que se refiere a las prácticas democráticas y republicanas. Tómense como ejemplos los casos de consejos municipales de políticas públicas compuestos por funcionarios públicos, como si fuesen representantes de la sociedad civil; o presupuestos participativos que consisten en presentar como decisiones populares medidas anteriormente decididas por el alcalde y llevadas a asambleas manipuladas o cooptadas, sólo para ser refrendadas. Dado el carácter previo (por ser el fenómeno reciente) y algunas veces precario (por las condiciones en las cuales ocurren) de los estudios, análisis y evaluaciones sobre los PPs y sobre los consejos, comentados en la sección anterior, hay que considerar como reales solamente los casos más significativos, sobre los cuales haya informaciones seguras. De esos se puede evaluar con más confianza la eficiencia, la eficacia, la efectividad y las posibilidades de permanencia y/o replicabilidad, así como la deseabilidad.

\subsection{La eficiencia, la eficacia y la efectividad de los PPs y de los CGMPP}

Se acepta aquí que la eficiencia es un concepto económico, que relaciona los resultados obtenidos a los recursos utilizados, estimando, de esa manera, la relación coste-beneficio (como se explica en los libros de texto de economía). El funcionamiento de los PPs y de las IPs implica costes, estos son los gastos en los que se incurre para realizar procesos de decisión colectiva que requieren divulgación, movilización, celebración de reuniones y eventos, trabajos de registro y control etc., además de los costes de oportunidad, de más difícil medición.

Para saber si el PP o una IP son eficientes, es necesario relacionar estos costes a los resultados producidos. Es prácticamente imposible hacer este cálculo con precisión, pues existen variables incluidas de difícil cuantificación, además de resultados intangibles o imperceptibles a corto plazo. Ejemplos: es difícil determinar que

apoyo, muchos titubearon cuando se les pidió que explicaran en qué consistía esa metodología de gestión de los recursos públicos y otros que estaban en posición de poder implementarlo, nunca lo hicieron.

8 A propósito, ver Wampler (2008), que identifica las diferencias de comportamiento en la implementación de innovaciones en la gestión presupuestaria local, relacionadas con los perfiles de los agentes 264 que las conducen: emprendedores de políticas públicas (alta actividad), defensores de políticas públicas (actividad limitada) y adoptantes formales (actividad mínima o nula). 
parte del tiempo de los empleados públicos (del departamento de hacienda o del gabinete del Alcalde, por ejemplo) implicados en el PP es coste de esta práctica innovadora y que parte es coste de las actividades cotidianas, son los mismos empleados públicos, ahora con responsabilidades adicionales, que no dedican necesariamente más tiempo a sus ocupaciones; no es posible, en la relección de un buen alcalde, separar los votos obtenidos por el mismo (derivados de su modo de dirigir y de relacionarse con los electores) de los votos conseguidos por haber puesto en marcha el Presupuesto Participativo en su primer gobierno; en qué medida la mejora en los indicadores de resultados de las políticas públicas compensa los costes de mantenimiento de estructuras y canales de decisión con participación ciudadana, pues aunque que haya una relación entre esos datos, no es fácil establecer la relación coste-beneficio.

La eficacia, a su vez, se define en este trabajo como indicador de la capacidad del PP o de una IP de alcanzar cierto objetivo o de cumplir una función definida. No se trata, en este caso, de medir numéricamente: la eficacia puede ser plena o total, media, baja o nula. Además, la eficacia no se define por sí, no es una relación matemática, como la eficiencia: ¿eficacia para qué? Es necesario preguntárselo siempre. Una experiencia de PP creada inicialmente para mejorar la gestión de los recursos financieros puede ser ineficaz para ello pero en cambio puede ser plenamente eficaz para dar cohesión al equipo de gobierno. Esto quiere decir que no se puede hablar de eficacia de los PPs y de las IPs en singular. Como esas innovaciones de gestión políticoadministrativas pueden tener impacto sobre numerosas variables, internas y externas a los gobiernos, hay que definir en los procedimientos y procesos de evaluación sobre cual o cuales de ellas se desea conocer la eficacia.

A pesar de las ya mencionadas, y a veces insuperables, dificultades para una plena percepción de la eficiencia y de la eficacia de los PPs y de las IPs, en la práctica se trabaja con evaluaciones aproximativas de esas características empíricas, relacionadas a casos específicos. Un cierto feeling acaba estableciéndose entre los participantes, asegurando la continuidad o no de las experiencias (excepto en el caso de las IPs legalmente exigidas, que se mantienen ignorando la eficiencia y la efectividad). Por ejemplo: después de dos o tres años experimentando el PP un gobierno "percibe" si las decisiones ciudadanas están costándole mucho o poco (en términos de recursos financieros, materiales y humanos y también en términos de apoyo electoral), y de la misma forma los ciudadanos que participan sienten, cuando los comparan a sus expectativas, si los esfuerzos que hacen les compensan o no.

La eficacia de los PPs y de las IPS, en ese caso, consiste en atender expectativas variadas, unas más y otras menos exigentes. La eficiencia se da cuando esas expectativas son mínimamente atendidas, a un coste mínimo. La ineficacia es, por definición, inadmisible, convirtiendo un PP o una IP en insostenible, por indeseable; la ineficiencia (alto coste comparado al valor de lo que se obtiene), a su vez, no es tenida en cuenta en ocasiones cuando hay un grado elevado de eficacia en el cumplimiento de las expectativas de algunos agentes con poder en el proceso. 
Como se puede deducir, entre eficiencia y eficacia existe una relación muy fuerte, siendo relativamente difícil separarlas para realizar una evaluación en experiencias concretas de PPs y de IPs. Aunque la verificación de la presencia de esas cualidades en los PPs y las IPs es, como se puede observar, de fundamental importancia.

La efectividad es la cualidad de producir efectos que las IPs pueden tener o no. Se confunde fácilmente con la eficacia. Para distinguirlas, conviene destacar que la eficacia está relacionada al resultado puro; mientras que la efectividad se refiere a la capacidad de las IPs de actuar, de funcionar (obteniendo o no los objetivos deseados). Es efectivo un PP que teniendo por objetivo deliberar sobre la mitad de las inversiones de un año, con la participación de los representantes de las distintas zonas de una ciudad, lo hace aunque esta deliberación sea, después, mal conducida por el gobierno, convirtiéndose por tanto en ineficaz. La participación ciudadana habrá sido efectiva pero sin eficacia para el presupuesto público.

La efectividad altera la realidad: algo que existía en potencia se materializa. Pero no la altera necesariamente en la dirección deseada, para lo que necesita la eficacia. La causa de la no efectividad-incapacidad de surtir efecto, por tanto puede incluso ser resultado de la baja eficiencia: insuficiente dotación de recursos para obtener el resultado deseado o la aplicación inadecuada de los recursos disponibles. Por ejemplo: un CGMPPs con suficiente número de participantes bien dispuestos y preparados para funcionar no tiene la consideración del titular de la materia sobre la cual le cabe deliberar y por eso no recibe las inversiones necesarias en infraestructuras y apoyo de personal para trabajar. Una relación coste-beneficio inadecuada (alto beneficio esperado y baja dotación de recursos), o sea, una ineficiencia, convierte al consejo en inefectivo, con consecuencias negativas también sobre su eficacia.

\subsection{La sostenibilidad de los PPs y los CGMPPs}

Para ser considerada como tal una institución necesita de un grado de permanencia, de aceptación y de uso pues en caso contrario no se incorpora a la realidad, al hacerlo sirve como referencia y funciona como guía de aglutinación de los actores. Sin sostenibilidad no existe evidentemente institución, aunque sea por un período limitado: hay instituciones más duraderas y otras menos duraderas.

La sostenibilidad inicial de una experiencia es condición sine qua non para que se institucionalice. Una ley puede convertir en obligatoria una experiencia y, de esa manera, contribuir para que ella se convierta en una institución. Puede ocurrir, al contrario, que una experiencia confirmada por el uso sea sacramentada legalmente. La segunda hipótesis consiste en un esfuerzo de ampliación y profundización de una institución, aumentando sus oportunidades de sustentación a lo largo del tiempo; la primera, es una tentativa de forzar la transformación de una experiencia en institución, que puede resultar negativa o positiva. O sea, la ley pura y simplemente no da 266 sostenibilidad a una institución ni la crea realmente, sobre todo si es una institución 
participativa, ya que la participación voluntaria -como ocurre en los PPs y las IPs- no se garantiza por decreto o ley.

La sostenibilidad de las experiencias de PPs y de IPs depende, con independencia de responder o no a exigencias legales, de un conjunto de factores endógenos y exógenos a ellas, incluyendo, entre otros, como ya se han identificado en los estudios recopilados y organizados por Avritzer y Navarro (2003) del diseño institucional, del compromiso político del gobierno, de las características de la sociedad civil, de la disponibilidad de recursos, de la experiencia acumulada y de la coyuntura política, económica, etc.

Las expectativas de los protagonistas e interesados, directa o indirectamente, en las experiencias participativas de procesos de decisión son también elementos determinantes de la sostenibilidad de estas experiencias, influyendo incluso en los ciclos alcistas y bajistas que algunas veces las caracterizan, debido a la influencia del ánimo o del desaliento que transmiten a los procesos y espacios de expresión y negociación. Además, los protagonistas e interesados establecen a su manera una percepción de la eficiencia, de la eficacia y de la efectividad de las IPs que puede contribuir o no a su sostenibilidad.

\subsection{La replicabilidad de los PPs y de los CGMPPs}

Las IPs que consiguen ser sostenibles, con buenos y difundidos resultados, se convierten con frecuencia en objeto de observación con el propósito de ser aplicadas en realidades distintas de las iniciales, fundamentalmente cuando su objetivo contiene aspectos comunes a situaciones y problemas de la misma naturaleza o aproximada a la de comunidades y organizaciones de diferentes lugares. Pasan entonces a ser replicadas, como ocurrió con el PP a partir de los años 1990, con el auxilio de varios centros nacionales e internacionales de investigación. La replicabilidad, sin embargo, requiere a veces adaptaciones de tal importancia que al final no se consigue, siendo en la realidad una IP que o no funciona o que no puede ser adecuadamente adoptada como la réplica, inicialmente deseada.

Exactamente igual pasa cuando la reproducción de la IP se convierte en obligatoria por ley como es el caso en Brasil de los CMGPPs, en este caso la replicabilidad puede ser cuestionada pues hacer que funcionen de la misma manera, con resultados mínimamente parecidos entre sí, los consejos en los 5.564 municipios brasileños es extremadamente difícil. Además, tales consejos se aplican a la realidad federativa brasileña, pero tal vez no a la argentina y pueden ser inimaginables en un país no federal, como Chile. El PP, a su vez, fue un fracaso en Buenos Aires (Peruzzoti, 2009), adquirió una fisionomía propia muy distinta de la brasileña en Italia (Alegretti, 2009), en España (Pineda, 2010; Pineda y Pires, 2011; Pineda y Pires, 2012), en Alemania (Herzberg, 2009) o en Portugal (Dias, 2009). Es necesario, por tanto, para conocer que se está replicando con éxito una IP ya testada y probada, comprobarlo empíricamente. 
Las principales preguntas que deben ser hechas, desde el punto de vista de la replicabilidad, son:

- Antes de la experiencia: ¿La IP es replicable en el nuevo contexto, por los nuevos actores? ¿Los nuevos actores son emprendedores de políticas públicas, defensores de las políticas públicas o adoptantes formales??

- Después de la experiencia o mientras ocurre: ¿Se trata, de hecho, de una réplica? ¿Con que grado de semejanza/precisión? Si hay diferencias entre la experiencia original y la actual, ¿estas fortalecen o debilitan la IP?

\subsection{La deseabilidad de los PPs y los CGMPPs}

La deseabilidad se refiere aquí no simplemente a la conveniencia de la puesta en marcha de una IP, sino a la percepción en la sociedad de esa conveniencia dentro de una visión específica del mundo. Aunque la claridad y la disposición para actuar son diferentes de un actor a otro (cada cual con objetivos y estrategias propias) en el conjunto de ellos existe una noción de relativo consenso (o de superioridad de una determinada correlación de fuerzas) que da como resultado cuando se intenta medirla (nunca numéricamente) una deseabilidad fuerte, media, frágil o no deseabilidad.

Hay dos maneras de considerar la deseabilidad: desde el punto de vista de un proponente, potencial adoptante o adoptante (que puede tener un potencial opositor o un efectivo opositor, o no); y desde el punto de vista de las visiones del mundo en sí, abstractamente consideradas. La distinción es relevante para delimitar los tipos posibles de evaluación bajo el enfoque de la deseabilidad.

Es posible evaluar la deseabilidad dentro de un contexto sociopolítico concreto, buscando identificar el nivel de deseabilidad o indeseabilidad de los actores implicados en el debate sobre implementar o evitar la implementación de una IP. Puede ocurrir, por ejemplo, que un gobierno local desee implantar el PP pero dentro de él haya sectores que aunque no se opongan claramente en la vida diaria crean dificultades para su funcionamiento o que haya segmentos ciudadanos favorables, pero que lo desean solamente hasta que sus intereses inmediatos sean atendidos (pavimentación de una calle, ampliación de un colegio). En estos casos, la evaluación dará una deseabilidad colectiva insuficiente aunque será difícil probarla empíricamente.

Es posible, por otro lado, evaluar la deseabilidad desde un punto de vista estrictamente teórico. Por ejemplo, colocando frente a frente, visiones de democracia en las cuales sean aceptadas las propuestas de protagonismo de no electos en las decisiones de los gobiernos y visiones de democracia que no aceptan esas propuestas. Vale, para ilustrar este tipo de análisis, enfrentar a los neorepublicanos y a los partida-

$9 \quad$ Ver nota 8. 
rios del public choice ${ }^{10}$. La teoría de la elección pública (TEP) o public choice da una explicación para el funcionamiento del gobierno basada en la lógica del comportamiento económico individual, racional-maximizante, típico de las relaciones mercantiles, contraponiendo a la visión del hombre público altruista (tantas veces invocada para justificar el uso del poder por medio del “contrato social”) la del hombre público egoísta, cuyo comportamiento interesado necesita ser controlado por mecanismos de castigo y recompensa (del mismo modo que ocurre con el agente económico concebido por el pensamiento liberal, de carácter hedonista y utilitarista), para que el resultado de su acción no sea nocivo para la sociedad. Los autores clásicos (Downs, 1957; Black, 1958; Buchanan y Tullock, 1962; Olson, 1965; Buchanam, 1973) y contemporáneos (por ejemplo, Niskanen 1971; Krueger, 1974; Nordhaus, 1975; Hibbs, 1977; Migué, 1997; Moe, 1997; Seldon, 1998) de esta corriente del pensamiento critican vehementemente la posibilidad de que individuos portadores de intereses personales y de grupos localizados actúen, de motu proprio, en la búsqueda del bien común o del interés colectivo, tal como se supone en algunas aproximaciones habituales en la Ciencia Política (identificadas por Macpherson, 1978) e igualmente de la Ciencia Económica (especialmente autores del desarrollo y defensores de mayores niveles de intervención estatal, como por ejemplo keynesianos y cepalinos).

Para los teóricos de la public choice el sistema electoral y los partidos cumplen, en la relación política entre representantes (electos) y representados (electores), un papel semejante al que el mercado y las empresas cumplen, respectivamente, en la relación económica entre ofertantes (empresas) y demandantes (consumidores). En la democracia representativa contemporánea, se deduce por esta teoría, hay una división del trabajo (típicamente weberiana) entre los políticos electos (detentores de cargos por medio de la legitimidad electoral), los burócratas (reclutados por méritos) y los electores, cada grupo especializado en el desarrollo de tareas específicas de los procesos de decisión y de provisión de bienes públicos. $Y$ esa división se reviste de una funcionalidad que garantiza los mejores resultados posibles, desde el momento en que exista una amplia difusión de información y los sistemas de castigo y recompensa funcionen en su interior adecuadamente. De esa forma, los costes de decisión y de acción, principalmente los primeros, son en teoría reducidos dramáticamente, haciendo económicamente irracional sistemas y procesos de elección pública y de acción colectiva que exijan protagonismo del ciudadano-elector más allá de la participación en procesos electorales periódicos ${ }^{11}$. De esa forma, metodologías de decisión presupuestaria pública que atribuyan poder de decisión a grupos de individuos que

10 Está en marcha un proyecto de investigación de uno de los autores de esta comunicación analizando la propuesta de participación ciudadana en las decisiones presupuestarias (presupuesto participativo) a partir de los enfoques del neorepublicanismo y del public choice. En esta sección el objetivo es solo llamar la atención sobre la posibilidad y la importancia de evaluar la deseabilidad de las IPs desde el punto de vista de teorías consagradas por las ciencias sociales, aunque sin tener en cuenta las experiencias en marcha, o sea, sin utilizar todavía elementos empíricos.

11 Olson (1965) afirma que la no participación en asuntos políticos es una elección racional del elector, en cuanto agente económico que tiene como objetivo la maximización de sus posiciones. Su argumentación sigue la lógica, presentada anteriormente por Condorcet en el siglo XVIII y por Benjamin Cons- 
no sean detentores de cargos políticos (tal como ocurre, por ejemplo, en las experiencias que a partir de finales de los años 80 se decidió llamar Presupuesto Participativo) son, en principio, contradictorias con la democracia representativa y, por encima de todo, económicamente indeseables.

Realmente el Presupuesto Participativo consiste, desde el punto de vista de la mayoría de sus defensores y proponentes, en una metodología de decisión presupuestaria que apunta en la dirección de una democracia más allá de la representativa liberal, en la cual los papeles de los actores implicados no son tan claros y esquemáticamente establecidos y divididos, como pretende la TEP. En la literatura sobre PPs se encuentran referencias a la democracia semidirecta (Vitale, 2005), a la democracia participativa (Souza Santos, 2002), a la democracia local (Abbers, 2000), a la democracia representativa comunitaria (Cabannes, 2005), a la democracia deliberativa (Bohman y Regh, 1997; Elster, 1998; Cohen, 2000), a la profundización de la democracia (Fung y Wright, 2001), a la democracia democratizada (Peruzzotti, 1998), a la democracia redistributiva (Souza Santos, 1998, 2002) etc. También los CMGPPs fomentan cambios, en la misma dirección de superación de la democracia representativa tradicional.

Así, los PPs y los CMGPPs son ideas, propuestas y experimentaciones que se mueven en el terreno de una concepción de la sociedad, del gobierno y de la política que al negar a la democracia representativa tradicional el carácter de la mejor forma de organización de las relaciones políticas, cuestiona, indirectamente, la validez de la teoría de la elección pública; y se aproxima más directamente al neorepublicanismo (Maihofer, 1990; Ackerman, 1993; Coats jr., 1994; Spitz, 1995; Goodwin, 1995; Veterli y Bryner, 1996; Sandel, 1996; Habermas, 1996; Terchek, 1997; Pettit, 1997; Giner, 1998; Onuf, 1998; Brugger, 1999; Skinner, 1999, 1998; Ihano, 1999; Berlanga, 1999), cuyas tesis se armonizan con la defensa de la profundización democrática de los regímenes políticos, por medio de una mayor participación ciudadana y en las cuales los PPs y los CMGPPs encajan con mayor facilidad. Y el presupuesto público, como las políticas públicas en cuanto instrumentos de decisión, son destacados focos de discusión en los que la contradicción y la confrontación entre la TEP y el neorepublicanismo pueden ser explorados, a fin de obtener una mejor comprensión acerca de los límites y potencialidades de experimentos innovadores para el trabajo de los gobiernos, en los cuales política y economía rivalizan y se tensionan mutuamente como fuente de explicaciones y justificaciones para los cambios institucionales existentes y futuros.

Si son evaluadas desde la perspectiva de la public choice (también conocida como teoría económica de la democracia), las IPs (principalmente los PPs y los CMGPPs concentrados en aspectos relativos al destino de los recursos) tienden a ser rechazados. Bajo la perspectiva neorepublicana, las IPs son deseables a priori, dado que su carácter político (al contrario del económico, como en el caso del public choice) es bastante posibilista: en ella el individuo no es simplemente un agente económi-

$270 \begin{aligned} & \text { tant en el siglo XIX, según la cual la existencia de representantes para } \\ & \text { lectivo libera a los individuos para cuidar de sus asuntos particulares. }\end{aligned}$ 
co, que se basa solo en la racionalidad mercantil maximizante; sino que está dotado de ciertos valores y es permeable a otros, teniendo la capacidad de implicarse en actividades de modo desinteresado (económicamente hablando) y disposición para destinar recursos (su tiempo, inclusive) para, colectivamente, buscar un modelo de relación social que considere más satisfactorio, en el cual el bien común prevalezca. Para los neorepublicanos, la democracia y el respeto a lo público tiene valor en si, debiendo ser preservados y profundizados. Ya que no son solamente instrumentos que garantizan la provisión de bienes públicos destinados a asegurar al ciudadanocontribuyente, soberano, la adecuada relación coste-beneficio entre los gastos en impuestos y los beneficios obtenidos con las políticas públicas.

Como se observa, subyace en cada una de las aproximaciones una visión del mundo y una concepción del hombre y de la sociedad casi opuesta, basada una en la ciencia económica y la otra en la ciencia política. Pero lo que consolida esos fundamentos no es ninguna de esas ciencias, sino filosofías políticas distintas. Y este hecho es una referencia que no se puede perder de vista cuando se tiene entre manos la tarea de evaluar experiencias de IPs, fundamentalmente cuando el objetivo perseguido es teorizar sobre ellas. Aunque se diga que no, hasta para sí mismo, cualquier evaluador o participante de procesos evaluativos de innovaciones como las IPs defiende una visión del mundo, del individuo y de la sociedad, en la cual la solución del conflicto inherente entre individuo y sociedad, entre interés público y privado, encuentra alternativas más individualistas o más colectivas o comunitarias. Incluso las cadenas de causalidad entre variables que se pretenden construir en las evaluaciones a partir de datos empíricos pueden ser contaminadas por este elemento, que condiciona la forma por la cual el conocimiento se construye, originándose en aquellos que son al mismo tiempo analistas y objetos de análisis. Y eso se refiere no sólo a las aludidas filosofías políticas, sino también a las distintas teorías del conocimiento, con respecto de las cuales, en esta comunicación no se puede más, dada la escasez de espacio para profundizar esta reflexión, que señalar la importancia de que no se sean olvidadas por los evaluadores de las IPs.

\section{UN ENFOQUE INTEGRADO PARA LA EVALUACIÓN DE LOS PPS Y LOS CGMPPS}

La evaluación de las experiencias concretas de IPs, como los PPs y los CGMPPs, es una tarea de gran complejidad, implicando dificultades conceptuales y metodológicas (Pires, R. R. C., 2011) e incluyendo muchas veces discusiones porque "La evaluación como forma de representación de una realidad implica la propia construcción de esta realidad a partir de un sistema específico de referencias, trayendo consigo un campo de significados y un conjunto de códigos que le son propios." (Campos, 2011, p. 57). Una aproximación integrada de esta evaluación, que considere los criterios abordados en la sección precedente, puede ser útil para enfrentarse a la complejidad y para administrar las dificultades y discusiones inherentes a los procesos evaluatorios de experiencias participativas. El diagrama 1 sintetiza esta aproximación, comentada a continuación. 
FIGURA 1

Visión esquemática integrada de los enfoques para la evaluación de las IPS

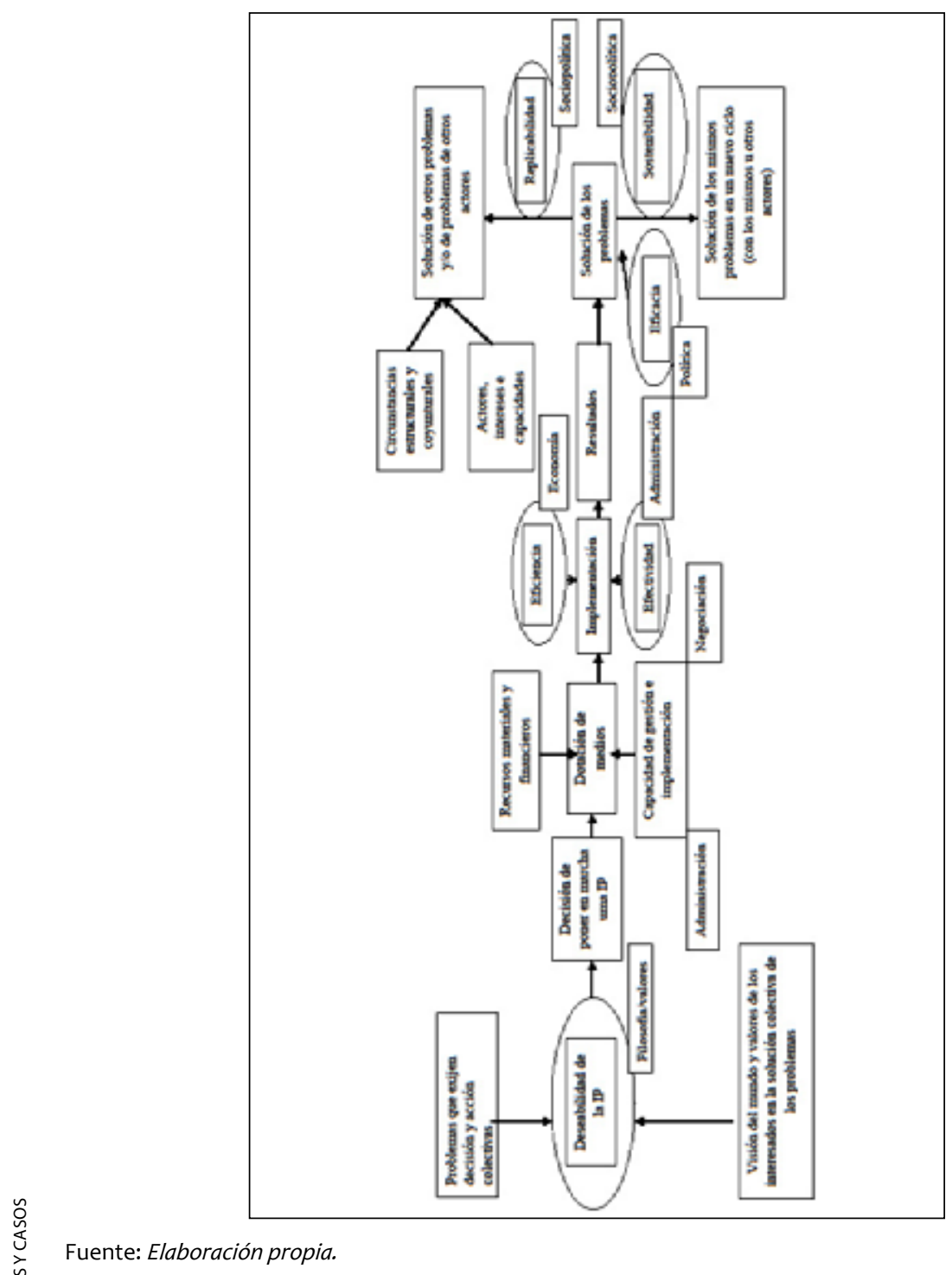

272 por ejemplo, puede concentrarse en el análisis de la replicabilidad, de la sostenibili- 
dad y de la eficacia, pues si resultaran negativas la decisión puede ser no intentar la implementación. Una evaluación estrictamente académica, por otra parte, podría comenzar por la deseabilidad, pues se puede estar interesado en los aspectos teóricos que llevan a la defensa de la democracia participativa en comparación a la democracia representativa tradicional.

En el diagrama se destaca el carácter multidisciplinar de la evaluación, con la presentación (por medio de figuras rectangulares que interrumpen las figuras ovales) de los ámbitos disciplinares en los que se sitúan los enfoques aludidos como necesarios en un trabajo evaluativo de mayor calidad: criterio de la eficiencia, ámbito de la economía; efectividad, administración; eficacia, política; sostenibilidad y replicabilidad, sociopolítica; deseabilidad, filosofía/ética/moral.

Obsérvese que el diagrama 1 presenta los criterios evaluativos de modo integrado, al conectarlos a momentos de un proceso de implementación de una IP. Este proceso se inicia cuando surge la voluntad (o deseo) de actores dispuestos a una solución dialogada/negociada de los problemas con los cuales se encuentran implicados y que exigen decisiones y acciones colectivas (enfoque de deseabilidad). La decisión para poner en marcha una IP se toma y los medios (recursos materiales y financieros y capacidades de gestión y negociación) para que sea implementada son movilizados. El manejo de los medios por los actores, con efectividad y/o eficiencia lleva a resultados, satisfactorios o no (criterios de efectividad y de eficiencia), que permiten, o no, solucionar los problemas y/o alcanzar los objetivos deseados con la creación de la IP (criterio de eficacia). Si los mismos modos de decidir y actuar pueden ser utilizados en otras circunstancias, por actores distintos de los originales, el enfoque de replicabilidad será posible evaluarlo. El enfoque de sostenibilidad es una condición necesaria de la replicabilidad (no se replica lo que de inicio no se mantuvo), la sostenibilidad se evalúa observando y analizando la continuidad de la IP durante más de un ciclo.

\section{CONCLUSIÓN}

La evaluación de las experiencias en marcha, durante las dos o tres últimas décadas, de PPs y de las diversas modalidades de IP (en Brasil, especialmente los CGMPPs) es una tarea que se hace indispensable ante la necesidad de pasar de la fase de acogida, por razones de fe y esperanza (en un contexto de redemocratización y reforma del Estado), a una nueva fase en las que puedan ser incorporadas al acervo de propuestas sistemáticamente fundamentadas y teóricamente sustentadas en una democracia más allá de la representativa tradicional o que, por el contrario, sean descartadas como tentativas insuficientes o, más aún, que sean clasificadas, por ahora, como experimentaciones de las cuales, por insuficiente sedimentación, no es posible, en la actual situación, sacar conclusiones definitivas o llegar a conclusiones provisionales con potencial para permitir o vislumbrar nuevos avances teóricos que permitan explicar las relaciones gobierno-sociedad en contextos democráticos contemporáneos. 
Con todo, no se debe perder de vista que, independientemente de los resultados de las evaluaciones sistemáticas en marcha y por hacer, la eclosión de esas IPs, en Brasil, a finales del siglo XX fue extremadamente importante para la recuperación y la consolidación de la democracia y aunque muchas veces sus objetivos precisos no hayan sido conseguidos crearon oportunidades y fueron instrumentos de la movilización social que cambió la apariencia política del país. En cuanto a su futuro, deben estar orientadas por las buenas evaluaciones que están siendo y serán hechas, ya que además dejaran de contar con un contexto social y político marcado por movilizaciones que pueden, por si solas (como en el pasado reciente), mantener el ímpetu innovador inicial de canales, mecanismos y formas de participación ciudadana en las decisiones de los gobiernos.

La literatura sobre las evaluaciones necesarias (sistemáticas y suficientemente imparciales, tanto como sea posible en las ciencias sociales) de los PPs y de las IPs, después de la euforia participativa de finales del siglo XX, comienza ya a ganar terreno, siendo las recopilaciones de Pires, R. R. C. (2011) y de Avritzer (2010) buenos ejemplos de esa tendencia. Lo que se pretende en este trabajo es contribuir, modestamente, a este debate, llamando la atención sobre dos aspectos esenciales de la evaluación de los PPs y de las IPs: primero, que toda evaluación que se haga debe proveerse, desde el principio, de suficiente claridad acerca de los distintos criterios posibles para evaluar los resultados y el funcionamiento de las IPs, eligiendo con cuidado uno o varios de estos criterios (y sus correspondientes metodologías), entendiendo que una evaluación que integre todos los criterios (tal como los hemos presentado en el diagrama 1 y explicado en la sección 4), es más consistente y completa; segundo, que la evaluación bajo el criterios de la deseabilidad es el único que permite la posibilidad de basarse en elementos estrictamente teóricos, debido a la existencia de teorías y escuelas de pensamiento (social, político y económico) que, por un lado, argumentan contra la participación ciudadana (o tienen una fuerte inclinación a oponerse a ella) en los procesos de decisión de los gobiernos (la public choice, por ejemplo) y teorías y escuelas que, por otro lado, hacen de la participación ciudadana una aspiración permanentemente perseguida para mejorar el funcionamiento del gobierno y su relación con la sociedad civil (el neorepublicanismo, por ejemplo).

En el debate teórico, que a final de cuentas, se convierte en filosófico y valorativo, independientemente del status científico del que disfruta, no se puede llegar a casi nada que pase más allá del campo de lo normativo, contaminado de ideas hegemónicas y contra hegemónicas; en cuanto a la teoría positiva, dentro de los límites de sus posibilidades en ciencias humanas, carece hasta el momento de muchos estudios (principalmente empíricos) para que se pueda elevar a las IPs a la condición de concepto enteramente integrado en el conocimiento ampliamente aceptado: la construcción de un nuevo paradigma teórico de democracia aún está en marcha en el campo de la ciencia, no obstante, por lo que parece, las experimentaciones se están acelerando en las últimas décadas, juntamente con el descrédito a las prácticas representativas convencionales y con el avance del discurso pro democracia participativa, 274 sea en el ámbito académico (como afirma la literatura de cuño neorepublicano), sea 
en el ámbito político, principalmente, como indican numerosos discursos y documentos institucionales (como, por ejemplo CLAD, 2009; OCDE, 2001).

Entre lo teórico y lo práctico, lo normativo y lo positivo, lo políticamente pragmático y lo analíticamente critico-reflexivo, se encuentra en estado de gaseoso a líquido el conocimiento científico sobre las instituciones participativas, sin que se sepa, hasta ahora, si se hará sólido y, de este modo, se unirá a los demás aparatos analíticos de las ciencias sociales, o terminará chocando con ellos o con algunos de ellos. Independientemente de lo que ocurra, las evaluaciones empíricas sobre el funcionamiento y los resultados de las IPs son necesarias, pero no se puede prescindir de los debates en el campo de los valores, de difícil abordaje cuando se utilizan metodologías de investigación cuantitativas y/o focalizadas exclusivamente en la búsqueda de vínculos de causalidad.

\section{BIBLIOGRAFÍA}

Abers, R. A. (2000): Inventing Local Democracy: Grossrooth Politics in Brazil. Boulder, Colorado: Lynne Rienner Publishers.

Ackerman, B. (1991): We the people: foundations. Cambridge, MA: Harvard University Press.

Alegretti, G. (2009): “Orçamentos Participativos na Itália: inovações dentro de um quadro em rápida transformação" en Silva, E.M. da; Cunha, E. S. M. (Org.). Experiências internacionais de participação. Belo Horizonte/São Paulo: UFMG/Ed. Cortez.

Avritzer, L. (2008): "Instituições participativas e desenho institucional”. Opinião Pública, v. 14, p. 43-64.

- (Org.) (2010): Experiências nacionais de participação. São Paulo: Cortez

- (2011): "A qualidade da democracia e a questão da efetividade da participação: mapeando o debate” en Pires, R. R. C. (Org.) (2011). Efetividade das instituições participativas no Brasil: estratégias de avaliação. Brasília: IPEA.

Avritzer, L.; Navarro, Z. (Org.) (2003): A inovação democrática no Brasil: o Orçamento Participativo. São Paulo: Cortez.

Avritzer, L.; Wampler, B. (2008): "The expansion of Participatory Budgeting in Brazil. An Analysis of the current cases based upon design and socio-economic indicators". (Research Report The World Bank/Municipal Development Program). Belo Horizonte: dic. 2008. Disponible en: <http://www.redeopbrasil.com.br/html/biblioteca/relatorio_banco_mundial_censo_op.pdf>. Acceso el: 20 enero de 2012.

Berlanga, J. L. V. (1999): Res Pública, Los Fundāmentos Normativos de la Politica. Madrid: Akal.

Black, D. (1958): The theory of commitees and election. Cambridge: Cambridge University Press.

Bohman, J. y Regh. W. (1997): Deliberative democracy: essays on reason and politics. Massachusetts: Massachesetts Institute of Tecnology.

Bresser-Pereira, L.C.; Wilheim, J.; Sola, L. (Org.) (1999). Sociedade e Estado em transformação. São Paulo/Brasília: Ed. UNESP/ENAP. 
Brugger, B. (1999). Republican Theory in Political Thouhgt, Virtous or Virtual?. Londres: MacMillan Press.

Buchanan, J. M. (1973): La hacienda pública en un proceso democrático. Madrid: Aguilar.

Buchanan, J. M.; Tullock, G. (1962): The Calculus of Consent. Ann Arbor: Universit of Michigan Press.

Cabannes, Y. (2005). “Presupuesto participativo y finanzas locales”. Porto Alegre, Programa de Gestión Urbana-ALC; UN-HABITAT; Alcaldía Municipal de Porto Alegre.

Campos, G. A. G. de (2011): "Participação e representações não avaliativas: a produção de significados nas experiências participativas" en Pires, R. R. C. (Org.) (2011). Efetividade das instituições participativas no Brasil: estratégias de avaliação. Brasília: IPEA.

Carvalho, A. I. (1995): Conselhos de saúde no Brasil: participação cidadã e controle social. Rio de Janeiro: Fase.

CLAD (2009): Carta Iberoamericana de Participación Ciudadana en la Gestión Pública. Caracas: CLAD. Disponible en:

<http://www.clad.org/documentos/declaraciones/carta-iberoamericana-de participacion-ciudadana>. Acceso el: 26 octubre de 2011.

Coats Jr., W. J. (1994): A Theory of Republican Character and Related Essays. Londres e Toronto: Susquehanna University Press.

Cohen, J. (2000): “Procedimiento y sustancia en la democracia deliberativa”. México, Metapolítica, v.4, n. 14, p.24-47, abr./jun.

Costa, D. (2010): "Vinte anos de Orçamento Participativo: análise das experiências em municípios brasileiros”. São Paulo, Cadernos Gestão Pública e Cidadania, v. 15, n. 56: p.8-28.

Dagnino, E. (Org.) (2002): Sociedade civil e espaços públicos no Brasil. São Paulo: Paz e Terra.

Dias, N. (2009): “Os Orçamentos Participativos em Portugal -uma nova dinamização na democracia local no país” en Silva, E.M. da; Cunha, E. S. M. (Org.). Experiências internacionais de participação. Belo Horizonte/São Paulo: UFMG/Ed. Cortez.

Downs, A. (1957): An economic theory of democracy. New York: Harper \& Row.

Elster, J. (1998). Deliberative democracy. Cambridge: Cambridge University Press.

Fung, A.; Wright, E. O. (2001): "Deepening democracy: inovation in empowered participatory governance". Politics \& Society, v. 29, n. 1, p. 5-41. March.

Giner, S. (1998): “ Las razones del republicanismo”, Claves de Razón Pratica, n. 81, pp. 2-13.

Gohn, M. da G. (2001): Conselhos Gestores e participação sociopolítica. São Paulo: Ed. Cortez.

Goodwin, C. S. (1995): A Resurrection of the Republican Ideal. Lanham, Mariland/ London: The University Press of America.

Guimarães, C. A. S. et. alt. (2009): Conselhos Gestores na Educação: perfil, discurso, funcionamento. Recife: FJN/Ed. Massangana.

Habermas, J. (1996): Between Facts and Norms, Contributions to a Discourse Theory of Law and Democracy. Cambridge, Ma.: The MIT Press.

Herzberg, C. (2009): Von der Bürger-zur Solidarkommune. Lokale Demokratie in Zei276 ten der Globalisierung. Hamburg: VSA-Verlag. 
Hibbs, D. A. (1977): "Political parties and macroeconomic policy”. American Political Science Review, n. 71, p. 1.467-1.487.

Krueger, A. (1974): "The political economy of the rent-seeking society". American Economic Review, n. 65, p. 191-303.

Llano, A. (1999): Humanismo Cívico. Madrid: Ariel.

Macpherson, C. B. (1978): A democracia liberal: origens e evolução. Rio de Janeiro: Zahar.

Maihofer, W. (1990): "The ethos of the republic and the morality of politics" en Bock, G.; Skinner, Q.; Viroli, M. (Eds.). Machiavelli and Republicanism. New York: Hill and Wang, 1999, pp. 283 y sgs.

Migué, J-L. (1997): "Public Choice in a Federal System”. Public Choice, v. 90, p. 235-254.

Moe, T. M. (1997): “The positive theory of public bureaucracy” en Mueller, D. (Ed.). Perspectives in Public Choice. Cambridge: Cambridge University Press.

Niskanen, W.A. (1971): Bureaucracy and Representative Government. Nueva York: Aldine-Athlerton.

Nordhaus, W. (1975): “The political business cycle”. Review of Economic Studies, n. 42, p. 169-90.

OCDE (2001): Citizens as partners: information, consultation and public participation in policy-making. Paris: OCDE.

Olson, M. (1965): The Logic of Collective Action: Public Goods and the Theory of Groups. Cambridge: Harvard University Press.

Onuf, N. G. (1998): The Republican Legacy in International Thought. Cambridge: Cambridge University Press.

Peruzzotti, E. (1998): Democratizing democracy: political culture, public sphere and coIlective learning in post-dictatorial Argentina. LASA Chicago, Illinois, Sept.

- (2009: "As políticas de inovação institucional: a implementação do Orçamento Participativo na cidade de Buenos Aires” en Silva, E.M. da; Cunha, E. S. M. (Org.). Experiências internacionais de participação. Belo Horizonte/São Paulo: UFMG/Ed. Cortez.

Pettit, P. (1997): Republicanism. A Theory of Freedom and Government. Oxford: Oxford University Press.

Pineda, C. (2010): “Los Presupuestos Participativos en España: un nuevo balance” en la Revista de Estudios de la Administración Local y Autonómica $n^{\circ}$ 311, INAP, Madrid, p. 277-303.

Pineda, C. y Pires, V. (2011): “Tipología de los Presupuestos Participativos (PPs) españoles” en el Encontro Nacional da Associação de Pós-Graduação e Pesquisa em Administração, 35, 2011, Rio de Janeiro. XXXV EnANPAD 2011. Anais: Rio de Janeiro, ANPAD.

Pineda, C. y Pires, V. (2012): “Características de las experiencias españolas de presupuesto participativo: intento de encuadramiento en una tipología”, Gestión y Análisis de Políticas Públicas, Nueva época $n^{\circ} 7$, enero-junio, INAP, Madrid.

Pires, R. R. C. (Org.) (2011): Efetividade das instituições participativas no Brasil: estratégias de avaliação. Brasília: IPEA.

Pires, V. (2001): Orçamento Participativo: o que é, para que serve, como se faz. Barueri: Ed. Manole. 
- (2009): "Transparência, participação e orçamento participativo: reflexões a partir do caso brasileiro".Temas de Administração Pública, v. 4, n. 5, 2009. Disponible en: http://master.fclar.unesp.br/Home/Departamentos/AdministracaoPublica/RevistaTemasdeAdministracaoPublica/pires5.pdf. Acceso el: 21 marzo de 2012.

Pires, V.; Martins, L. de J. (2011): “Orçamento Participativo (OP) após vinte anos de experiências no Brasil: mais qualidade na gestão orçamentária municipal?" en el Encontro Nacional da Associação de Pós-Graduação e Pesquisa em Administração, 35, 2011, Rio de Janeiro. XXXV EnANPAD 2011. Anais: Rio de Janeiro, ANPAD.

Pires, V.; Silva, R. G. da (2004): “Orçamento Participativo: a opinião de políticos do interior paulista em 1999”. APG-PUCSP Revista Acadêmica Multitemática, São Paulo, ano XI, n. 30, p. 315-323.

Ribeiro, A. C. T. y Grazia, G. de (2003): Experiências de Orçamento Participativo no Brasil. Período de 1997 a 2000. Petrópolis: Ed. Vozes.

Sandel, M. (1996): Democracy's Discontent, America in Search of a Public Philosophy. Cambridge, Londres, The Belknap Press of Harvard University Press.

Seldon, A. (1998): The dilemma of democracy: the political economics of over-government. London: Institute of Economic Affairs (Hobart Paper, n. 136).

Skinner, Q. (1998): Liberty before Liberalism. Cambridge: Cambridge University Press. Skinner, Q. (1999): “The republican ideal of political liberty” en Bock, G.; Skinner, Q.; Viroli, M. (Eds.). Machiavelli and Republicanism. New York: Hill and Wang, pp. 293 e segs.

Souza Santos, B. de (2002): Democratizar a democracia: os caminhos da democracia participativa. Rio de Janeiro: Civilização Brasileira.

- (1998): "Budgetings in Porto Alegre: Towards a Redistributive Democracy". Politics and Society, v. 26, n. 4, p. 461-510.

Spitz, J. F. (1995): La liberté politique. Paris: PUF.

Tatagiba, L. (2002): “Os Conselhos Gestores e a democratização das políticas públicas no Brasip” en Dagnino, E. (Org.). Sociedade civil e espaços públicos no Brasil. São Paulo: Paz e Terra.

Terchek, R. (1997): Republican Paradoxes and Liberal Anxieties.Lanham: Boulder; Londres: Rowman and Littlefield.

Tullock, G.; Seldon, A.; Brady, G. (2005): Falhas de Governo: uma introdução à teoría da escolha pública. Rio de Janeiro: Instituto Liberal.

Vaz, A. C. N. (2011): "Da participação à qualidade da deliberação em fóruns públicos: o itinerario da literatura sobre conselhos no Brasil” en Pires, R. R. C. (Org.) (2011). Efetividade das instituições participativas no Brasil: estratégias de avaliação. Brasília: IPEA.

Veterli, R. y Bryner, G. (1996): In Search of the Republics, Public Virtue and the Roots of the American Government. Lanham: Roman and Littlefield.

Vitale, D. (2005): “Democratizando o processo orçamentário: a experiencia do Orçamento Participativo nos municipios brasileiros" en Brasil, Secretaria do Tesouro Nacional. Finanças Públicas. IX Prêmio Tesouro Nacional: Monografias. Brasília: ESAF. 